FSU-SCRI-97-128

UW-PT-97-26

\title{
Probing the Region of Massless Quarks in Quenched Lattice QCD using Wilson Fermions
}

\author{
Robert G. Edwards, Urs M. Heller, Rajamani Narayanan \\ SCRI, The Florida State University, Tallahassee, FL 32306-4130, USA \\ Robert L. Singleton Jr. \\ Department of Physics, University of Washington, Box 351560, Seattle, WA \\ 98195-1560, USA
}

\begin{abstract}
We study the spectrum of $\mathrm{H}(m)=\gamma_{5} \mathrm{~W}(-m)$ with $\mathrm{W}(m)$ being the Wilson-Dirac operator on the lattice with bare mass equal to $m$. The background gauge fields are generated using the $\mathrm{SU}(3)$ Wilson action at $\beta=5.7$ on an $8^{3} \times 16$ lattice. We find evidence that the spectrum of $\mathrm{H}(m)$ is gapless for $1.02<m<2.0$, implying that the physical quark is massless in this whole region.
\end{abstract}

PACS \#: 11.15.Ha.

Key Words: Lattice QCD, Wilson fermions.

\section{Introduction}

The conventional wisdom obtained from lattice simulations of QCD using Wilson fermions at various values of gauge couplings $\beta$ and bare quark mass $m$ is that there is a critical line $m_{c}(\beta)$ where the physical quark is massless. This critical line is not universal, since different definitions for what one means by massless quark give different results for $m_{c}(\beta)$ at finite $\beta$ because the lattice theory has artifacts that only vanish in the continuum limit. One method is to define $m_{c}(\beta)$ as the line where the pion mass is zero. Such a definition can only be implemented by extrapolating from values of $m$ where the pion mass is substantially away from zero. In dynamical simulations, critical slowing down makes it hard to generate enough independent configurations to obtain a good 
estimate of the pion mass when it is small. In quenched simulations, there is no problem of critical slowing down in the generation of gauge field configurations, but it becomes difficult to compute quark propagators below a certain pion mass due to the presence of small eigenvalues in the spectrum of the WilsonDirac operator. Thus, as already mentioned, one relies on an extrapolation to estimate $m_{c}(\beta)$ using data for pion masses greater than $0.5 m_{\rho}$ [1].

The chiral symmetry of QCD with massless quarks is expected to be spontaneously broken. In the continuum, the spectral density $\rho(\lambda)$ of $\gamma_{5} \not D$ is symmetric, and spontaneous breakdown of chiral symmetry is due to a non-zero value for $\rho(0)$ [2]. Furthermore, gauge fields with nontrivial topology will result in exact zero eigenvalues of $\not D$ with a specific chirality [3]. To study the spontaneous breakdown of chiral symmetry on the lattice using Wilson fermions, and also to compute the topological charge of the gauge fields using Wilson fermions as a probe, we consider $\mathrm{H}(m)=\gamma_{5} \mathrm{~W}(-m)$, where $\mathrm{W}(m)$ is the standard Wilson-Dirac operator with bare mass equal to $m$. The operator $\mathrm{H}(m)$ can be viewed as the Hamiltonian describing the evolution of a four dimensional fermion in a fifth direction. The evolution is in a gauge field background that is independent of the fifth direction, and this Hamiltonian plays a central role in the definition of the chiral determinant 国. Level crossings in the spectrum of $\mathrm{H}(m)$ as a function of $m$ correspond to the presence of topological objects in the background gauge field configuration with the net level crossing being the topological charge of the gauge field [4. Level crossings at finite values of $\beta$ will not occur at a single value of $m$, but will instead occur over a region in $m$ [5]. This is expected to be the case in both pure gauge theories and QCD where the fermion dynamics is included. As such, the spectrum of $\mathrm{H}(m)$ will not have a gap in a region $m_{1}(\beta)<m<m_{2}(\beta)$. In this region, the spectral density in the thermodynamic limit is expected to have a finite density of eigenvalues at zero leading to a spontaneous breakdown of chiral symmetry [6]. It is natural to say that the physical quark mass is zero in the whole region $m_{1}(\beta)<m<m_{2}(\beta)$. The existence of a region as opposed to a single point is due to finite lattice spacing, and we expect both $m_{1}(\beta)$ and $m_{2}(\beta)$ to go to zero as $\beta$ goes to infinity. Therefore, one does not have a critical line $m_{c}(\beta)$ where the physical quark is massless. Instead, one has a region in the $(\beta, m)$ plane bounded by the lines $m_{1}(\beta)$ and $m_{2}(\beta)$ where the physical quark is massless. The critical line $m_{c}(\beta)$ obtained from the extrapolation of pion masses measured in the region $m<m_{1}(\beta)$ will most likely lie in the region between $m_{1}(\beta)$ and $m_{2}(\beta)$, but it need not coincide with $m_{1}(\beta)$. If $m_{c}(\beta)$ is the only point where the physical quark mass is zero, then we have a region of bare quark masses between $m_{1}(\beta)$ and $m_{c}(\beta)$ where the physical quark mass is positive but the spectrum does not have a gap. Because of low lying eigenvalues in the region $m_{1}(\beta)<m<m_{c}(\beta)$, it is difficult to measure the propagators of mesons. Low lying eigenvalues make it numerically difficult to compute the propagator, and even if one succeeds in computing the inverse, violent fluctuations in the propagator over the en- 
semble of gauge fields make it prohibitive to get a good statistical estimate of the propagator. Therefore, in the region $m_{1}(\beta)<m<m_{c}(\beta)$, one cannot extract the pion mass by conventional means and show that it is non-zero. A method has been recently invented to remove the low lying eigenvalues in the region $m_{1}(\beta)<m<m_{c}(\beta)$ [7] under the assumption that the low lying eigenvalues in the above region are lattice artifacts. This can be thought of as a non-local modification of the Wilson-Dirac operator; however, it might enable one to compute the pion mass in the region $m_{1}(\beta)<m<m_{c}(\beta)$. Gauge field configurations with different physical properties are responsible for the low lying eigenvalues at different values of $m$ and all these gauge field configurations in the ensemble are equally important and collectively give rise to the existence of the chiral condensate. Therefore, it is not appropriate to remove the low lying eigenvalues in the region of $m_{1}(\beta)<m<m_{c}(\beta)$. Instead, we take the point of view that the physical quark is massless in the whole region $m_{1}(\beta)<m<m_{2}(\beta)$, which shrinks to the point $m=0$ as $\beta$ goes to infinity.

Our aim in this paper is to present some evidence in support of the picture outlined in the previous paragraph. To this end, we focus on pure gauge field configurations generated using the $\mathrm{SU}(3)$ Wilson action at $\beta=5.7$ on an $8^{3} \times 16$ lattice. Using an ensemble of 50 configurations, we will show that $\mathrm{H}(m)$ is gapless in the mass range between $m_{1}=1.02$ and $m_{2}=2.0$, indicating that the physical quark is massless in this whole region. We do not consider $m>2$ since this is a region of $m$ which is clearly unphysical in the continuum limit. 1 Using the method proposed in Ref [6], we will show that chiral symmetry is spontaneously broken in this whole region of $m$. For the quenched approximation on a $16^{3} \times 32$ lattice at $\beta=5.7$, the data of Ref. [8] at $m<1$ yield a an extrapolated value of $m_{c}=1.047$ where the pion mass becomes zero. This is inside the gapless region. We will show that the pion propagator for a given gauge field background can be estimated quite well in this region by using the spectral representation for $\mathrm{H}(m)$ and restricting the spectral sum to the low lying eigenvalues. This will enable us to compute the pion propagator more efficiently than with conventional means since we can sum over all possible source points on the lattice and effectively restore translational invariance. The resulting propagator, when averaged over an ensemble of configurations, will still have large fluctuations due to the variation in the magnitude of the low lying eigenvalues. But close to $m_{1}=1.02$ the fluctuations will not be large, and we will see that the pion propagator in the region from $m_{1}$ to $m_{c}$ is not described by an exponential decay but rather by a power law, providing further evidence that the physical quark described by the Wilson-Dirac fermion is massless in this region. A little more physical insight

\footnotetext{
${ }^{1}$ In fact, $m>1$ does not yield a unitary theory in the continuum limit, but we are, however, away from the continuum limit here. At $m=2$ the free fermion has four poles in the propagator due to "doublers", and since we are interested in the gap in the spectrum of the $\mathrm{H}(m)$ we avoid the region of $m>2$.
} 
on the properties of the gauge fields responsible for the low lying eigenvalues will be gained by looking at the eigenvectors of $\mathrm{H}(\mathrm{m})$ corresponding to these low lying eigenvalues. In particular, the eigenvectors corresponding to zero eigenvalues of $\mathrm{H}(m)$ have a natural association with localized objects on the lattice, and the eigenvectors themselves should be localized. In the sample of 50 configurations, we have nine such zero eigenvalues of $\mathrm{H}(m)$ for $m<1.047$. We will show that the corresponding eigenvectors are all localized. The zero spatial momentum component of these eigenvectors has a large extent in time, occupying anywhere from seven to ten lattice spacings. A detailed study of the level crossing over the whole range of $m$ from $m=1$ to $m=2$ yields information about the topological content of the gauge field, including the size distribution of the topological objects on the lattice. The analysis of the size distribution shows that larger objects cross at smaller values of $m$, indicating that the crossings at $m<m_{c}$ should indeed correspond to larger objects than the ones associated with crossings at $m>m_{c}$.

This paper is organized as follows. In the next section we review the connection between the level crossings of $\mathrm{H}(m)$ and topology. We also review the method to measure the chiral condensate using a term that breaks parity [6]. This method measures the chiral condensate arsing from spontaneous breaking only and gives a zero result unless the spectrum is gapless. In section 3, we measure the chiral condensate using the spectral representation of $\mathrm{H}(m)$ and show that there is a non-zero chiral condensate in the whole region from $m_{1}=1.02$ to $m_{2}=2.0$. We also obtain the spectral density as a function of $m$ and show that the gap closes at $m_{1}=1.02$, and we find that it remains closed for all higher values of $m$ up to the largest value we studied, namely $m_{2}=2$. This is consistent with a non-vanishing chiral condensate in this whole region of $\mathrm{m}$. In section 4 , we focus on the region from $m_{1}=1.02$ to $m_{c}=1.047$. In this region we compare the shape of the eigenvectors very close to the crossings points with the pion propagators at the same value of $m$. We find excellent agreement, indicating that the pion propagator is dominated by that particular eigenvector. By employing the spectral representation of $\mathrm{H}(m)$ and taking a restricted spectral sum, we show that one can obtain the pion propagator quite well over a region around the crossing point. We then use the restricted spectral sum to compute the average pion propagator over the entire ensemble and show that the resulting pion propagator is not described by an exponential decay. Our conclusions are presented in section 5 . 


\section{Properties of the spectral flow of $\mathrm{H}(m)$}

We start this section by writing down the central quantity in the paper, namely the Wilson-Dirac Hermitian Hamiltonian $\mathrm{H}(m)$ on the lattice in a fixed gauge field background. Upon suppressing the dependence on the $\mathrm{SU}(3)$ background gauge field $U, \mathrm{H}(m)$ in the chiral basis is

$$
\mathrm{H}(m)=\left(\begin{array}{cc}
\mathrm{B}-m & \mathrm{C} \\
\mathrm{C}^{\dagger} & -\mathrm{B}+m
\end{array}\right) ; \quad 0 \leq m \leq 2,
$$

where

$$
\begin{aligned}
& \mathrm{C}_{i \alpha, j \beta}\left(k, k^{\prime}\right)=\frac{1}{2} \sum_{\mu} \sigma_{\mu}^{\alpha \beta}\left[U_{\mu}^{i j}(k) \delta_{k^{\prime}, k+\hat{\mu}}-\left(U_{\mu}^{\dagger}\right)^{i j}\left(k^{\prime}\right) \delta_{k, k^{\prime}+\hat{\mu}}\right] \\
& \mathrm{B}_{i \alpha, j \beta}\left(k, k^{\prime}\right)=\frac{1}{2} \delta_{\alpha, \beta} \sum_{\mu}\left[2 \delta_{i j} \delta_{k k^{\prime}}-U_{\mu}^{i j}(k) \delta_{k^{\prime}, k+\hat{\mu}}-\left(U_{\mu}^{\dagger}\right)^{i j}\left(k^{\prime}\right) \delta_{k, k^{\prime}+\hat{\mu}}\right] \\
& \sigma_{1}=\left(\begin{array}{cc}
0 & 1 \\
1 & 0
\end{array}\right) ; \quad \sigma_{2}=\left(\begin{array}{cc}
0 & -i \\
i & 0
\end{array}\right) ; \quad \sigma_{3}=\left(\begin{array}{cc}
1 & 0 \\
0 & -1
\end{array}\right) ; \quad \sigma_{4}=\left(\begin{array}{cc}
i & 0 \\
0 & i
\end{array}\right),
\end{aligned}
$$

and where $k$ and $k^{\prime}$ label the lattice sites, $\alpha$ and $\beta$ are two component spinor indices, and $i$ and $j$ are three component color indices.

The fermionic action in terms of $\mathrm{H}(m)$ is

$$
S_{f}=\psi^{\prime} \mathrm{H}(m) \psi
$$

with $\psi^{\prime}$ related to the standard $\bar{\psi}$ by $\bar{\psi}=\psi^{\prime} \gamma_{5}$. The fields $\psi^{\prime}$ and $\psi$ are independent Grassmann variables. The choice for the sign of $m$ differs from the standard one. In the free theory $(U=1)$, the action with $-m$ describes a free fermion with mass equal to $m$.

The operator $\mathrm{H}(m)$ can also be viewed as the single particle Hamiltonian describing the propagation of a fermionic field in the fifth direction under a background gauge field that is static in that direction. The many-body Hamiltonian describing the fermionic evolution in the fifth direction is

$$
\mathcal{H}(m)=a^{\dagger} \mathrm{H}(m) a
$$

where $a$ and $a^{\dagger}$ are canonical fermion annihilation and creation operators for

Dirac fermions. The chiral determinant on the lattice, which is the result of integrating out a single Weyl fermion in a fixed gauge background, is given by the overlap [4]

$$
\operatorname{det} \mathrm{C} \equiv\langle 0-\mid 0+\rangle \text {, }
$$


where $|0 \pm\rangle$ are the ground states of $\mathcal{H}( \pm m)$ for some fixed value of $m$ in the range $(0,2)$. All choices of $m$ in this range give rise to the same chiral theory in the continuum limit but will have different lattice spacing effects away from the continuum.

Equation (7) along with (6) provides an immediate connection between level crossings in the spectral flow of $\mathrm{H}(m)$ as a function of $m$ and the global topology of the background gauge field [ [4]. On a finite lattice containing $V$ sites, $\mathrm{H}(\mathrm{m})$ is a $2 K \times 2 K$ matrix with $K=6 \mathrm{~V}$. The ground states $|0 \pm\rangle$ are obtained by filling all the negative energy states of $\mathrm{H}( \pm m)$. For an arbitrary gauge field background, one can rigorously show that $\mathrm{H}(-m)$ always has $K$ negative energy states for all values of $m>0$. Consider a background gauge field for which $\mathrm{H}(m)$ has $(K-Q)$ negative eigenvalues with $Q>0$. Then $\operatorname{det} \mathrm{C}$ as defined in (7) is zero and $\left\langle 0-\left|a_{1}^{\dagger} \cdots a_{Q}^{\dagger}\right| 0+\right\rangle$ is non-zero, implying that $Q$ fermions of a fixed chirality are created in this gauge background. Such a gauge field configuration on the lattice will then be said to carry a topological charge of $Q$ in analogy with the continuum index theorem [3]. Examples of such gauge field configurations can be constructed on the lattice [4]. Since $\mathrm{H}(-m)$ has $K$ negative energy states, while $\mathrm{H}(m)$ has $(K-Q)$ negative energy states, the spectral flow of $\mathrm{H}(m)$ as a function of $m$ should have a net level crossing of $Q$ (from below zero to above zero) as one goes from $-m$ to $m$. Therefore $\mathrm{H}(m)$ should have at least $Q$ zero eigenvalues, and these will generically occur at $Q$ different values of $m$. Typically, $n_{+}$levels will cross from below zero to above zero, and $n_{-}$levels will cross from above zero to below zero, with the topological charge being given by $Q=n_{+}-n_{-}$. Then $\mathrm{H}(m)$ will have $\left(n_{+}+n_{-}\right)$ zero eigenvalues at $\left(n_{+}+n_{-}\right)$different values of $m$.

It is instructive to write down the zero eigenvalue equation of $\mathrm{H}(m)$ using (11):

$$
(\mathrm{B}-m) u+\mathrm{C} v=0 ; \quad \mathrm{C}^{\dagger} u-(\mathrm{B}-m) v=0 ; \quad u^{\dagger} u+v^{\dagger} v=1 .
$$

Trivial manipulation of the above equation results in the following necessary condition for a zero eigenvalue [4]

$$
u^{\dagger} \mathrm{B} u+v^{\dagger} \mathrm{B} v=m
$$

Since B, defined in (3), is positive for all values of the background gauge field, it follows that zero eigenvalues occur only for $m>0$ [4]. As one takes a lattice gauge field and interpolates to the continuum, all solutions to (8) that occurred at some value of $m>0$ will now occur at $m=0$, since the norm of $\mathrm{B}$ on any state goes to zero in the continuum. Level crossings on the lattice will occur at different values of $m$ because the eigenvectors associated with the level crossings have a typical size inside which they are localized. Eigenvectors with larger sizes will vary slower across the lattice than eigenvectors with smaller sizes, implying that $m$ as given in (9) will be bigger for smaller size objects. Since we expect topological objects of varying sizes in an ensemble of gauge 
fields generated at some finite gauge coupling on the lattice, we expect level crossings to occur in a region of $m$.

Gauge fields generated at a finite gauge coupling on the lattice have a finite correlation length in lattice units. One can define the spectral distribution of $\mathrm{H}(m)$ in the thermodynamic limit, in which the number of lattice sites becomes large, as the one obtained by considering a large ensemble of gauge field configurations generated on a lattice with all linear sizes greater than the correlation length. The spectrum of $\mathrm{H}(m)$ corresponding to each gauge field configuration in the ensemble will be discrete, but the spectral distribution for an infinite ensemble is expected to be continuous. We denote the continuous spectral distribution by $\rho(\lambda ; m)$. This will be an even function of $\lambda$ since for every eigenvalue $\lambda$ of $\mathrm{H}(m)$ in a fixed gauge field configuration there is an eigenvalue $-\lambda$ for the Hamiltonian associated with the parity transformed gauge field on the lattice $\mathrm{B}$. If $\rho(0 ; m)$ is non-zero, then $\mathrm{H}(m)$ does not have a gap at that value of $m$. Low lying eigenvalues of $\mathrm{H}(m)$ and level crossings in $\mathrm{H}(m)$ can give rise to a non-zero value for $\rho(0 ; m)$. One can establish a non-zero chiral condensate if $\rho(0 ; m)$ is non-zero by adding a term $i h \psi^{\prime} \psi$ to the fermionic action in (5) [6]. In the presence of this term,

$$
i\left\langle\psi^{\prime} \psi\right\rangle_{U}=\frac{1}{2 K} \sum_{n} \frac{h}{\lambda_{n}^{2}(m)+h^{2}}
$$

in a fixed gauge field background $U$, where $2 K$ is the total number of degrees of freedom. The right hand side is obtained by taking the average over $U$ and its parity transformed partner. To obtain the chiral condensate, one has to compute the ensemble average in the limit of infinite volume at finite values of $h$ and extrapolate to the limit of vanishing $h$. In this limit, we obtain a connection between the chiral condensate and the non-zero value of $\rho(0 ; m)$ [6], namely,

$$
i\left\langle\psi^{\prime} \psi\right\rangle=\pi \rho(0 ; m)
$$

Note that a non-zero value for $i\left\langle\psi^{\prime} \psi\right\rangle$ comes solely from $\rho(0 ; m)$. This is the advantage of measuring the chiral condensate using $i\left\langle\psi^{\prime} \psi\right\rangle$. Existence of the chiral condensate can be obtained by an explicit computation of (10) over an ensemble of configurations as a function of $h$.

\section{Evidence for a chiral condensate over a region of $m$}

We generated 50 independent $\mathrm{SU}(3)$ gauge field configurations on an $8^{3} \times 16$ lattice at $\beta=5.7$ using the standard single plaquette Wilson action. We

\footnotetext{
${ }^{2}$ Lemma 4.4 in Ref 沟 provides a proof of this statement.
} 


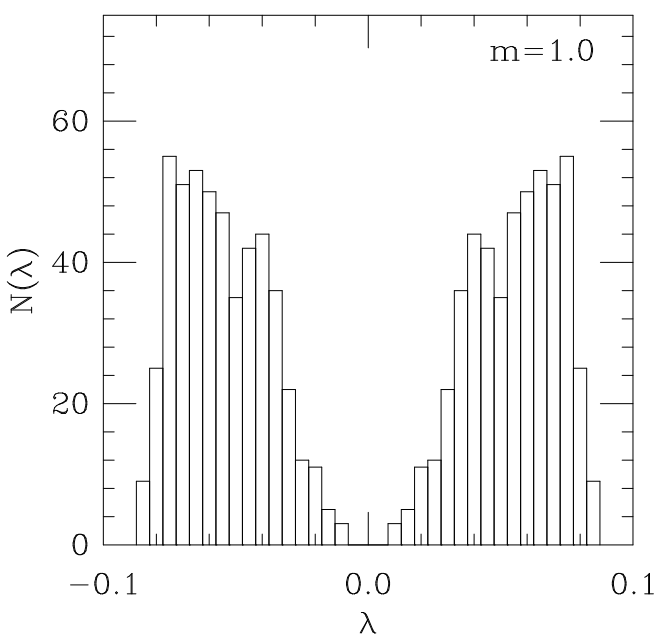

Fig. 1. Spectral distribution of $\mathrm{H}(m)$ at $m=1$ obtained from the ten eigenvalues closest to zero on 50 gauge field configurations at $\beta=5.7$. The bin width is 0.005 .

computed the ten eigenvalues of $\mathrm{H}(m)$ closest to zero as a function of $m$. For this, we used the Ritz functional method developed in Ref. [9] and improved for the case of multiple eigenvectors in Ref. [10]. The Ritz functional method finds the lowest eigenvalues of $\mathrm{H}^{2}(m)$, and these are in fact the eigenvalues of $\mathrm{H}(m)$ closest to zero. The ten eigenvalues of $\mathrm{H}^{2}(m)$ were obtained to a relative precision of $10^{-5}$ or to an absolute precision of $10^{-9}$ depending on which condition is satisfied first in the iterative procedure. Only ten eigenvectors were kept in the iterative procedure and were reorthogonalized every ten iterations. Eigenvalues and eigenvectors were obtained for masses between $m=1$ to $m=2$ with increments of $\Delta m=0.02$. In first order perturbation theory

$$
\frac{d \lambda_{n}(m)}{d m}=-\phi_{n}^{\dagger}(m) \gamma_{5} \phi_{n}(m),
$$

with $\phi_{n}(m)$ being the eigenvector corresponding to $\lambda_{n}(m)$. The mass increment was sufficiently small to allow for an accurate interpolation of eigenvalues between consecutive values of $m$. The ten eigenvalues closest to zero varied in the range from -0.1 to 0.1 as a function of $m$ over the entire ensemble. Figure [ shows the spectral distribution $\rho(\lambda ; m)$ at $m=1$. This distribution has a gap and the physical quark is massive. Figure 2 shows the spectral distribution $\rho(\lambda ; m)$ at $m=1.03$. Here the spectrum does not have a gap and the physical quark is massless.

Since $\rho(0 ; 1.03)$ is non-zero, we expect a chiral condensate at $m=1.03$. To investigate the chiral condensate as a function of $m$, we use (I0) to measure 


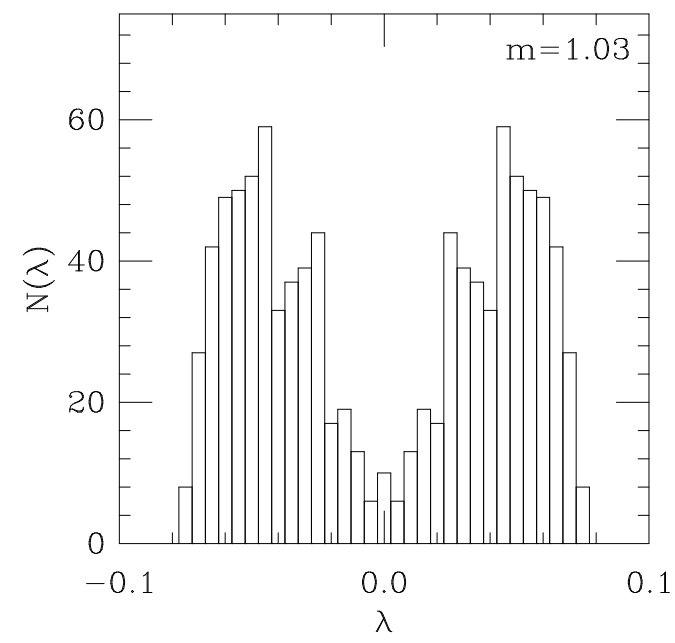

Fig. 2. Spectral distribution of $\mathrm{H}(m)$ at $m=1.03$ obtained from the ten eigenvalues closest to zero on 50 gauge field configurations at $\beta=5.7$. The bin width is 0.005 .

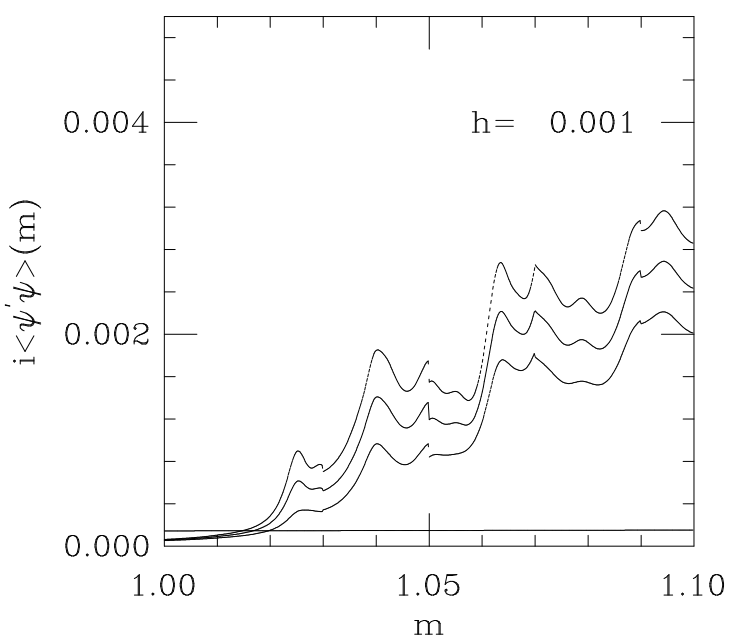

Fig. 3. The chiral condensate $i\left\langle\psi^{\prime} \psi\right\rangle(m)$ from $m=1.0$ to $m=1.1$ with $h=0.001$. The middle curve is the estimate of the chiral condensate, and the curves on either side show the error of this estimate. The solid line at the bottom is the result for free fermions. 


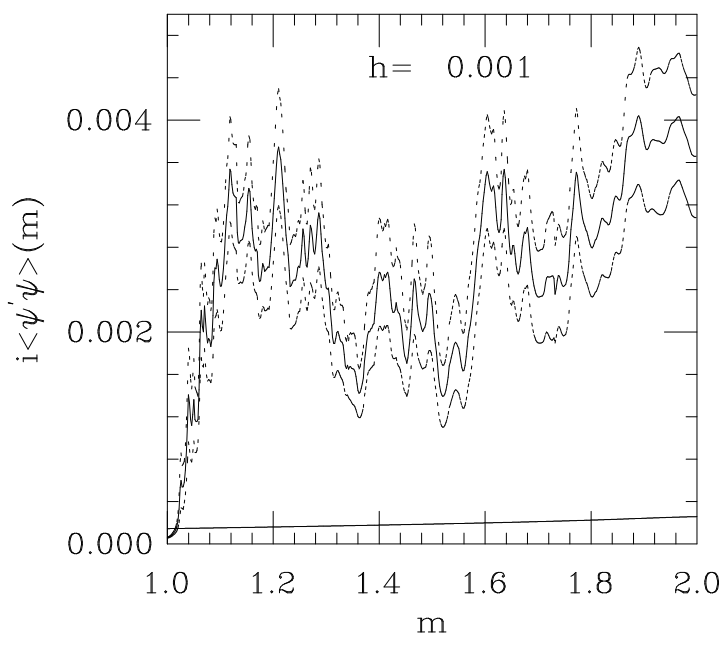

Fig. 4 . The chiral condensate $i\left\langle\psi^{\prime} \psi\right\rangle(m)$ from $m=1.0$ to $m=2.0$ with $h=0.001$. The middle curve is the estimate of the chiral condensate, and the curves on either side show the error of this estimate. The solid line at the bottom is the result for free fermions.

$i\left\langle\psi^{\prime} \psi\right\rangle_{U}$ per configuration and then compute an ensemble average to obtain $i\left\langle\psi^{\prime} \psi\right\rangle$. For small values of $h$ in (10), only the low lying eigenvalues will contribute. Setting $h=0.001$ in (10) and summing over the ten lowest eigenvalues of $\mathrm{H}(m)$, we obtain a value for $i\left\langle\psi^{\prime} \psi\right\rangle(m)$ as a function of $m$. We also compute $i\left\langle\psi^{\prime} \psi\right\rangle(m)$ for free fermions at $h=0.001$ by summing over the whole spectrum. We plot the result in the range $1 \leq m \leq 1.1$ in Fig. 3. The solid line at the bottom of the figure is the result for free fermions. When $m>1.02$, the result from the gauge theory is significantly larger than from free fermions, and is consistent with a non-zero chiral condensate in this mass region. Figure. 国illustrates the behavior of the condensate $i\left\langle\psi^{\prime} \psi\right\rangle(m)$ for the region $1.0<m<2.0$. This result is consistent with a non-zero value for $\rho(0 ; m)$ for the entire range of masses between $m_{1}=1.02$ and $m_{2}=2.0$. Note that the critical quark mass $m_{c}=1.047$, obtained by extrapolating the pion mass to zero, is inside the region where the spectrum is gapless. To understand the dependence of the chiral condensate on the parameter $h$, in Fig. 5 we plot $i\left\langle\psi^{\prime} \psi\right\rangle$ versus $h$ at a typical mass $m=1.5$. The picture is basically the same at other values of $m$ greated than $m_{1}$. Clearly there is a wide region of $h$ where the condensate is basically flat, in contrast to the free fermion result which drops exponentially in the plot with a logarithmic scale. As expected from (10), the condensate goes to zero as $h$ goes to zero and as $h$ goes to infinity. The errors at the peak region are large due to the large fluctuations in $\lambda_{n}^{-2}$ coming from the low lying eigenvalues. If the spectral sum is restricted to the six lowest eigenvalues, as opposed to the ten lowest, all the above results are essentially unaltered. 


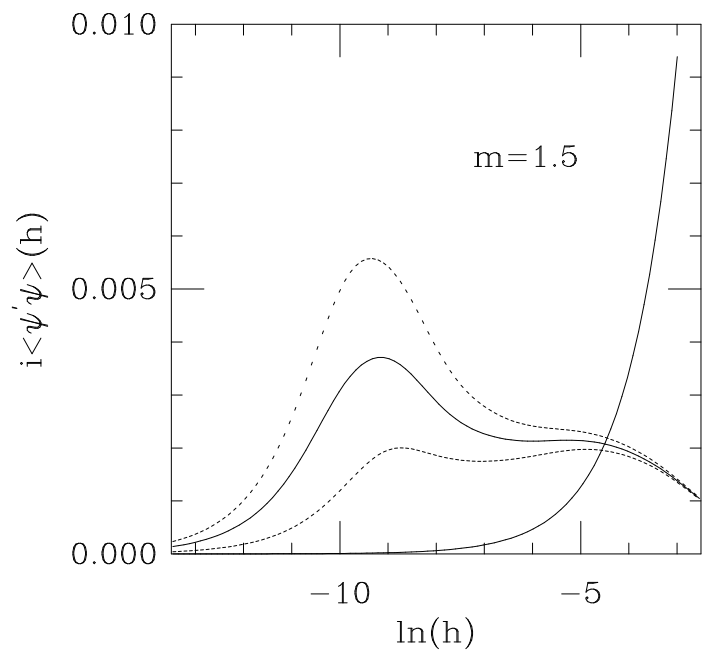

Fig. 5. Chiral condensate $i\left\langle\psi^{\prime} \psi\right\rangle(h)$ at $m=1.5$ viewed as a function of $h$. The $\mathrm{x}$-axis is logarithmic to show a wide range in $h$. The middle curve with a peak is the estimate of the chiral condensate, and the curves on either side show the error of this estimate. The exponentially varying solid line is the result for free fermions.

\section{The mass range $m_{1}<m<m_{c}$}

In the previous section we explored the consequences of the low lying eigenvalues of $\mathrm{H}(m)$. We showed that the spectrum is gapless in a mass range bounded by $m_{1}=1.02$ and $m_{2}=2.0$, and we provided evidence for a non-vanishing chiral condensate in the gapless region. Our interpretation is that the physical quark is massless in this whole region of $m$. If this is indeed the case, we should find zero eigenvalues of $\mathrm{H}(\mathrm{m})$ scattered throughout this region due to the presence of topological objects in the gauge field configurations. Equation (8) defines the zero eigenvalue equation for $\mathrm{H}(m)$, and (9) is a necessary condition for a zero eigenvalue. Based on (9), it was argued in section 2 that larger topological objects will result in a zero eigenvalue at smaller values of $m$. In this section we first look at the sizes of the topological objects and their crossing points, and we present evidence supporting the above statement. Then we explore the region $m_{1}<m<m_{c}$, where $m_{c}=1.047$ is the point at which the extrapolated pion mass vanishes, to establish the following points:

- Eigenvectors associated with zero eigenvalues of $\mathrm{H}(m)$ in the $m_{1}<m<m_{c}$ region correspond to large localized objects extending roughly over half the lattice in the long direction. 
- The pion propagator for a fixed gauge field background with level crossings, at values of $m$ close to a crossing point, is well described by a spectral sum restricted to a few low lying eigenvalues of $\mathrm{H}(m)$.

- The ensemble average of the pion propagator obtained by a restricted spectral sum at $m_{1}=1.02$ do not fit an exponential decay. Instead they fit a power law.

To this end we write down the spectral representations of $\mathrm{H}(m)$ and $\mathrm{H}^{-1}(m)$. The eigenvalue equation for $\mathrm{H}(m)$ is

$$
\sum_{k^{\prime} b j} \mathrm{H}_{a i, b j}\left(k, k^{\prime}\right) \phi_{b j}^{n}\left(k^{\prime}\right)=\lambda_{n} \phi_{a i}^{n}(k),
$$

and we have dropped the dependence on $m$ in the above equation. Here $k$ and $k^{\prime}$ label the sites on the lattice, $a$ and $b$ are four component spinor indices, $i$ and $j$ are three component color indices, and $n$ labels the eigenvalues. The eigenvectors are normalized to yield $\sum_{k a i}\left[\phi_{a i}^{m}(k)\right]^{*} \phi_{a i}^{n}(k)=\delta_{n m}$. In terms of the eigenvalues and eigenvectors, the spectral representations of $\mathrm{H}$ and $\mathrm{H}^{-1}$ are

$$
\begin{aligned}
\mathrm{H}_{a i, b j}\left(k, k^{\prime}\right) & =\sum_{n} \lambda_{n} \phi_{a i}^{n}(k)\left[\phi_{b j}^{n}\left(k^{\prime}\right)\right]^{*} \\
\mathrm{H}_{a i, b j}^{-1}\left(k, k^{\prime}\right) & =\sum_{n} \frac{1}{\lambda_{n}} \phi_{a i}^{n}(k)\left[\phi_{b j}^{n}\left(k^{\prime}\right)\right]^{*} .
\end{aligned}
$$

The point-like pion field is given by

$$
\pi(k)=\sum_{a i} \psi_{a i}^{\prime}(k) \psi_{a i}(k),
$$

and using the spectral representation of $\mathrm{H}^{-1}$ in (15), the pion propagator in a fixed gauge field background becomes

$$
\left\langle\pi(k) \pi\left(k^{\prime}\right)\right\rangle_{U}=\sum_{n m} \frac{1}{\lambda_{n} \lambda_{m}} f_{n m}(k) f_{n m}^{*}\left(k^{\prime}\right),
$$

where the gauge invariant functions $f_{n m}(k)$ are defined by

$$
f_{n m}(k)=\sum_{a i}\left[\phi_{a i}^{n}(k)\right]^{*} \phi_{a i}^{m}(k) .
$$

The eigenvalues of $H(m)$ will sweep out a spectral flow as $m$ varies over its range, and typically some levels will cross, thereby producing a set of zero modes at discrete values of the mass. Let us examine such a zero mode, $\phi_{a i}^{0}(k)$, at some crossing point $m$. In what follows, it is convenient to consider the zero momentum part of $f_{00}(k)$ defined by $f(t)=\sum_{\vec{k}} f_{00}(\vec{k}, t)$. We can associate a size with $f(t)$ using the pion correlator

$$
C_{0}(t)=\sum_{t^{\prime}} f\left(t^{\prime}\right) f\left(t+t^{\prime}\right)
$$




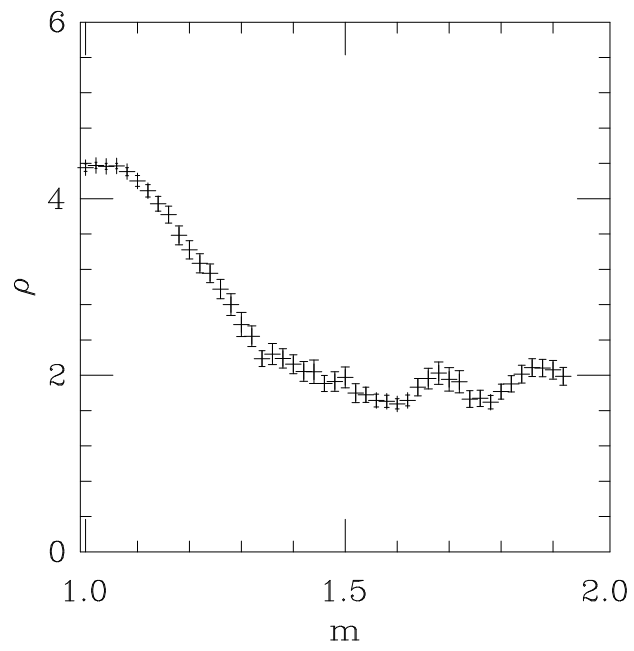

Fig. 6. The localization size as a function of the crossing point. The data point at $m$ includes all crossings that occurred in the interval $m \pm 0.05$.

which is a periodic function of $t$ with a peak at $t=0$. We define the localization size, $\rho$, through the second moment of $C_{0}(t)$ :

$$
\rho^{2}=\frac{\sum_{t=-7}^{8} t^{2} C_{0}(t)}{\sum_{t=-7}^{8} C_{0}(t)} .
$$

Recall that the lattice has 16 sites in the time direction, and in calculating the localization size we order them to run from -7 to 8 . Figure 6 shows the localization size of the zero modes as a function of the location of the crossing point. The trend in this figure is consistent with the picture that localized objects of a larger size cross at smaller values of $m$.

We now focus on crossings in the region between $m_{1}$ and $m_{c}$, i.e. the mass range $1.02<m<1.047$. In our ensemble of 50 configurations, we found seven which contained one crossing each within this mass range, and one configuration with two crossings between $m_{1}$ and $m_{c}$. For all nine zero modes, $f(t)$ peaks at some value of $t$ in the range $0 \leq t \leq 15$, and in Fig. 7 we have plotted $f(t)$ for two of them. In numerically solving for the eigenvalues of $H(m)$, we generically find near zero-modes since we sweep over discrete values of the mass parameter. The solid line in Fig. 7 corresponds to a mode whose eigenvalue was closest to zero along the computationally obtained discrete spectral flow. It occurred at a mass of $m=1.04$, and using (12) we find that the associated zero crossing happens at $m_{0}=1.0375$, which is very close indeed. As one can see, the mode peaks somewhere between $t=8$ and $t=9$ and has a width of about seven or eight lattice spacings. The dashed line is the eigenvector for another 


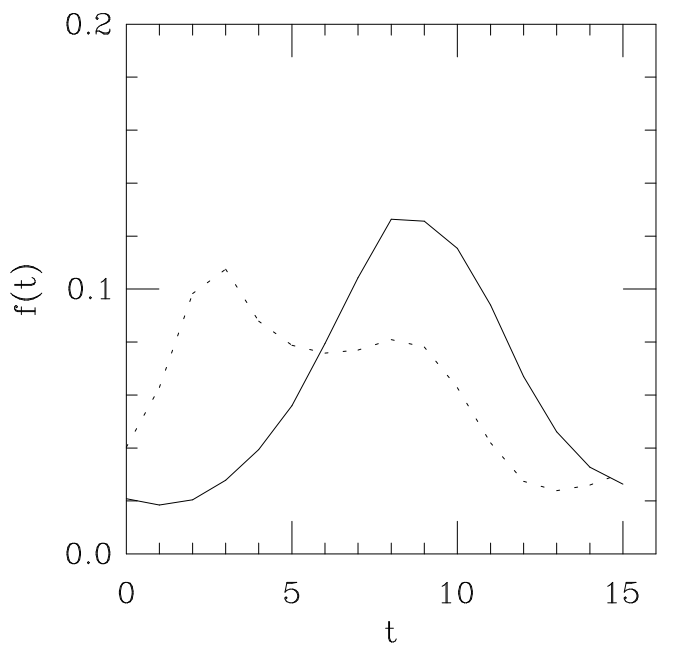

Fig. 7. The function $f(t)$ for two near zero-modes within the mass range $m_{1}<m<m_{c}$. The solid line corresponds to a mode at $m=1.04$ for a certain configuration within the ensemble, while the dashed line corresponds to a mode at $m=1.02$ for a different gauge field configuration.

configuration in the ensemble whose eigenvalue along the discrete spectral flow is again closest to zero. It occurs at $m=1.02$, and (12) implies that the associated zero crossing is at $m_{0}=1.025$. This mode has a double peak structure, and it stretches over ten lattice spacings. Of the nine zero modes within the range $m_{1}<m<m_{c}$, seven of them had a single peak in $f(t)$ while two exhibited double peaks, and all them stretched over seven to ten lattice spacings.

We also looked at $f_{00}(k)$ over the whole lattice and found that the modes were localized in all four directions but were not spherical. These modes are large objects having widths of seven to ten lattice spacings in the long direction and widths of six or so in the short directions. The finite size of the lattice plays a significant role in the shape of the mode. Furthermore, the associated lattice gauge field backgrounds of these eigenvectors are far from the continuum limit, and as such are not smooth configurations. This also affects the shape of these modes.

Near values of $m$ where $\mathrm{H}(m)$ has a zero eigenvalue, we expect the spectral decomposition of $\mathrm{H}^{-1}(m)$, as given by (15), to be dominated by the low lying modes. We test this on the configuration corresponding to the solid line in Fig. 7 at two mass points $m=1.029$ and $m=1.036$, the latter being close to the crossing point $m_{0}=1.0375$. We shall restrict the spectral sum in (17) to 


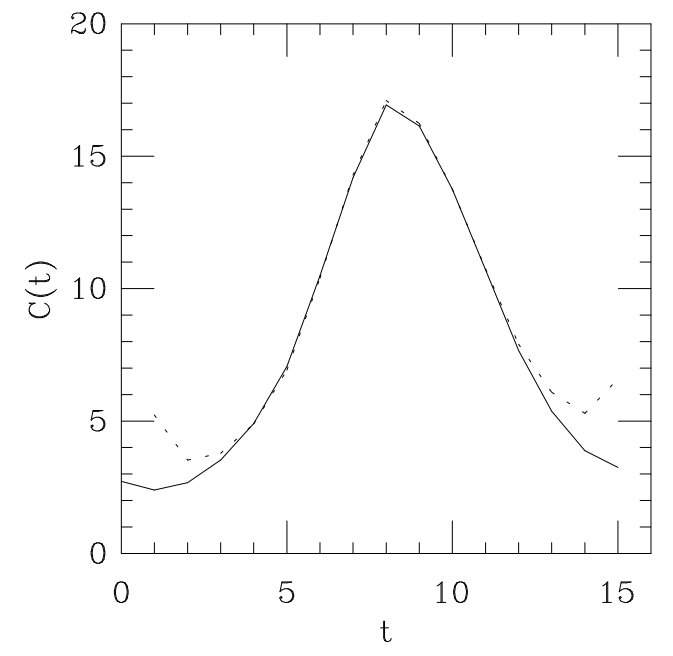

Fig. 8. Comparison between the full pion propagator (dashed) and the pion propagator obtained from the restricted spectral sum (solid), both of which are in the fixed gauge field background corresponding to the solid line of Fig. 7 . The mass is $m=1.029$, which is near the crossing value $m_{0}=1.0375$.

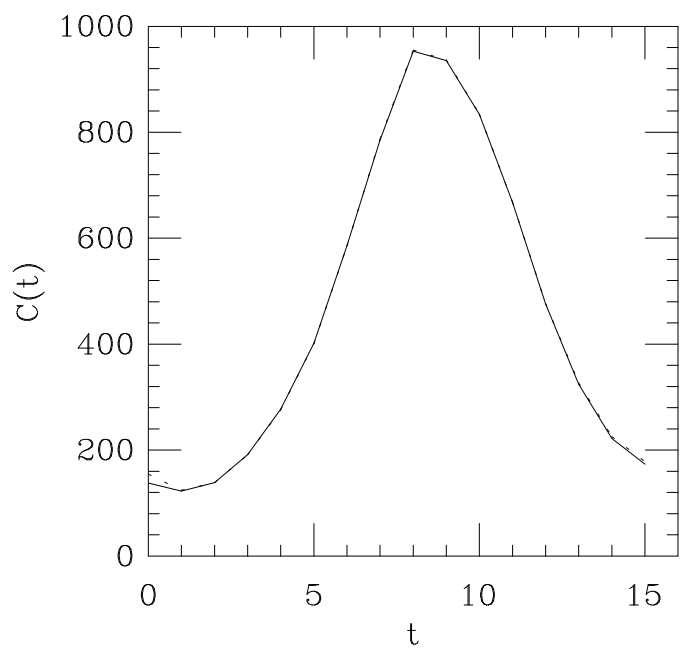

Fig. 9. Comparison between the full pion propagator (dashed) and the pion propagator obtained from the restricted spectral sum (solid) using the same gauge field background as in Fig. 8. This time the mass is $m=1.036$, which is even closer to the crossing value $m_{0}=1.0375$, and the two curves lie almost on top of one another. 
run only over the ten eigenvalues closest to zero, and we compute the quantity $C(t)=\sum_{\vec{k}}\langle\pi(0) \pi(\vec{k}, t)\rangle_{U}$ using both the restricted spectral sum and the full propagator obtained by inverting $\mathrm{H}(m)$ with standard techniques. Fig. 8 shows $C(t)$ at $m=1.029$ and Fig. 9 illustrates a comparison at $m=1.036$. At $m=1.029$ both methods agree for $3 \leq t \leq 12$, while at $m=1.036$ they agree at all values of $t$, which is an expected result since the latter mass is closer to the crossing point. Furthermore, it should come as no surprise that the pion propagator itself, $C(t)$, looks very much like $f(t)$ for the corresponding zero mode, in this case the solid line of Fig. 7 .

The pion propagators of Figs. 8 and 9 are far away from an expected exponential decay starting at $t=0$, and instead they have a dominant peak around $t=8$. Such configurations are the reason why one cannot measure the pion mass at values of $m$ in the region between $m_{1}=1.02$ and $m_{c}=1.047$, since they cause large statistical fluctuations. This has been known for quite some time and was first observed in Ref. [11]. As previously mentioned, the peak is due to a zero of $\mathrm{H}(m)$ close to values of $m$ where the pion propagator is being measured. Since we have presented evidence that the pion propagator can be well approximated by a restricted spectral sum, one can reduce the statistical fluctuations by computing the pion propagator as an average over all possible translations of the background gauge field. This can easily be done in the restricted spectral sum, but it would be prohibitive if one were using conventional methods to compute the pion propagator, since one would have to do a volume worth of inversions of the Wilson-Dirac operator. It should be mentioned that a restricted spectral sum has been used to study the spectroscopy in the region where $\mathrm{H}(m)$ has a gap [12]. The average pion propagator over all possible translations of a fixed gauge field background is

$$
C_{\mathrm{av}}(k)=\frac{1}{V} \sum_{k^{\prime}}\left\langle\pi\left(k^{\prime}\right) \pi\left(k+k^{\prime}\right)\right\rangle,
$$

and we find it useful to study $C^{\prime}(t)=\sum_{\vec{k}} C_{a v}(\vec{k}, t)$. In Fig. 10 we plot $C^{\prime}(t)$ at $m=1.029$ for the same configuration as in Fig. 8. This function has the expected behavior for a pion correlator in the sense that it falls off starting at $t=0$, but it does not fit an exponential decay. Instead, it fits a power law of the form obtained using the zero mode solution in the presence of a classical instanton. The spherically symmetric zero mode associated with a classical instanton take the form 13

$$
\psi^{\dagger}(x) \psi(x) \sim \frac{1}{\left(r^{2}+\rho^{2}\right)^{3}},
$$

which gives a contribution to the pion correlator of

$$
C_{\mathrm{cl}}(x)=\int \frac{d^{4} y}{(2 \pi)^{4}} \frac{1}{\left(y^{2}+\rho^{2}\right)^{3}} \frac{1}{\left((x+y)^{2}+\rho^{2}\right)^{3}} .
$$




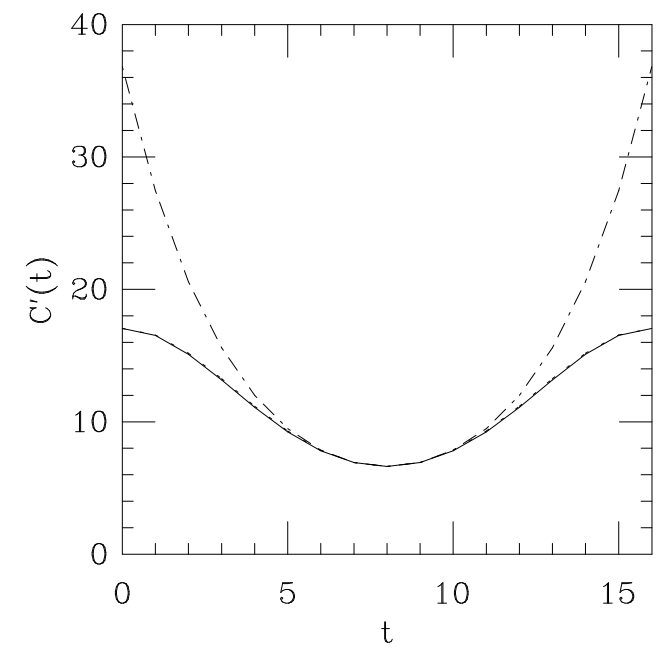

Fig. 10. The solid line is the pion propagator of Fig. 8 averaged over all possible translations of the gauge field. The dotted line that falls on top of the solid line is a fit to a power law, and the dashed line shows an exponential fall off in comparison.

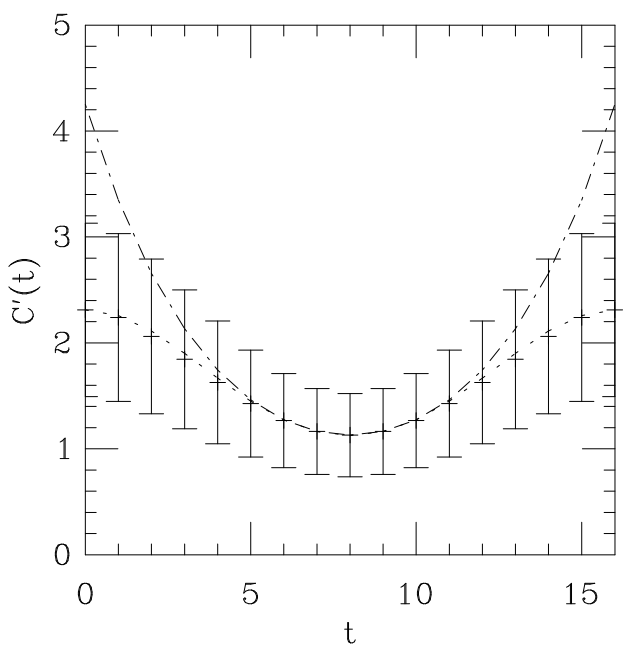

Fig. 11. The data points with error bars show the pion correlator $C^{\prime}(t)$ averaged over the ensemble of 50 configurations. The dotted line is a power law decay and the dashed line shows an exponential fall off in comparison. 
The above integral can be evaluated and the resulting functional form can be fitted to $C^{\prime}(t)$. The fit corresponding to $\rho=5.1375$ is shown as the dotted line in Fig. 10, which falls right on top of $C^{\prime}(t)$ represented by the solid line. In contrast to this fit, the dashed line shows the behavior of $C\left(e^{-\alpha t}+e^{-\alpha(16-t)}\right)$ with $\alpha=0.3$. Note that $C^{\prime}(t)$ is below the exponential decay near $t=0$ implying that the decay is slower at $t=0$ when compared to the decay at $t=8$. This is atypical behavior for the correlator since excited states would result in a faster decay at $t=0$. This is another indication that the behavior is inconsistent with an exponential decay. The power law behavior is consistent with a non-vanishing chiral condensate in the region $1.02<m<1.047$, as found in the previous section. Furthermore, Fig. 7 shows that the zero eigenvalues in the above region of $m$ are caused by large topological objects extending over several lattice spacings. Therefore, zero eigenvalues are caused by physical objects in the background gauge field, which results in a non-vanishing chiral condensate and a power law behavior in the pion mass. The proposal of Ref. [7] shifts the position of these zeros to some value of $m$ greater than $m_{c}=1.047$, thereby removing all low lying eigenvalues in the region $m<m_{c}$. This should also make the chiral condensate vanish for $m<m_{c}$ and remove the power law contribution to the pion propagator in this region. This indicates that the proposal in Ref. [7] is a non-local change to the Wilson-Dirac operator motivated by the need for a single value of $m$ where the pion mass vanishes. The proposal of Ref. [7] does nothing to the zero crossings that occur at $m>m_{c}$, and therefore one will still have a region where $\mathrm{H}(m)$ is gapless. In this region there will be a non-vanishing chiral condensate and the pion will be massless. In effect, the modification of the eigenvalues proposed in Ref. [7] slightly changes the region where the spectrum of $\mathrm{H}(m)$ is gapless and we see no compelling reason to make this non-local modification to the Wilson-Dirac operator.

We now proceed to compute $C^{\prime}(t)$ as an average over the whole ensemble at $m_{1}=1.02$. At this mass value there is evidence for a non-zero density of eigenvalues at zero, as presented is section 3. Figure 11] shows the result of averaging over the whole ensemble. The errors, although large, still enable us reasonably estimate of the pion correlator at this value of $m$. The dotted line shows the same power law function as the one used in Fig. 10 with $\rho=5.675$, whereas the dashed line shows an exponential decay with $\alpha=0.25$. Again we see the same behavior as in Fig. 10, namely the data is below the exponential decay at $t=0$. The pion propagator at different times is correlated and it is interesting to note that the power law fit goes through all the central values. The propagator fits the power law better and is not consistent with an exponential decay. We have therefore shown that it is not appropriate to alter the low lying eigenvalues in the region of $\mathrm{m}$ between $m_{1}=1.02$ and $m_{c}=1.047$. We have also established that (21) is a powerful method for estimating the pion propagator in this region using the restricted spectral sum to compute the right hand side of this equation. 


\section{Conclusions}

We have studied the spectrum of $\mathrm{H}(m)=\gamma_{5} \mathrm{~W}(-m)$, with $\mathrm{W}(m)$ being the Wilson-Dirac operator on the lattice with a bare mass $m$. We generated 50 pure $S U(3)$ gauge configurations using the single plaquette Wilson action at $\beta=5.7$ on an $8^{3} \times 16$ lattice. We found that the spectrum is consistent with it being gapless in the region between $m_{1}=1.02$ and $m_{2}=2.0$. Using a parity breaking operator as proposed in Ref. [6], we estimated the chiral condensate and found it to be non-zero in this whole region. Therefore, it is natural to say that the physical quark is massless in this region. This is in contrast to declaring a single value, $m_{c}=1.047$, where the pion mass obtained by extrapolation from the region $m<1$ vanishes [8], as the unique point where the physical quark is massless. We computed the pion correlator using a restricted spectral sum and by averaging over all possible translations of the background gauge field. We found that the pion correlator is consistent with a power law decay at $m_{1}=1.02$, with the power law coming from the low lying eigenvalues of $\mathrm{H}(\mathrm{m})$. This further confirms that the physical quark is massless in this whole region of $m$, as opposed to a single point. We also showed that the eigenvectors corresponding to zero eigenvalues of $\mathrm{H}(m)$ in the region between $m_{1}=1.02$ and $m_{c}=1.047$ are localized and roughly span half the lattice in the long direction (about $1.36 \mathrm{fm}$ ). As such, the low lying eigenvalues in this region are associated with physical objects in the gauge field background.

The region in $m$ where the physical quark is massless shrinks to the single point $m=0$ in the continuum limit. But the region has a finite width if $\beta$ is finite. The width will change if we improve the Wilson-Dirac operator. We studied the spectral flow for a few gauge field configurations using the SheikholeslamiWohlert [14 improvement scheme. We found that the gap closes at a smaller value of $m$, but preliminary indications are that it remains closed all the way up to $m=2$, as in the Wilson-Dirac case. For the Wilson-Dirac operator, the width of the gapless region is not expected to change as we go to infinite lattice volume at fixed $\beta$. But we expect more low lying eigenvalues as we go to larger lattice volume since the chiral condensate arises from spontaneous symmetry breaking, and the effect should become stronger in larger lattice volume. For the same reason, one should see more zero eigenvalues of $\mathrm{H}(\mathrm{m})$ in a fixed region of $m$. An investigation of ten configurations on a $16^{3} \times 32$ lattice at $\beta=5.7$ showed that there were seven zero eigenvalues in the region $1.02<m<1.047$. This is a greater percentage than the nine zero eigenvalues found in 50 configurations on the $8^{3} \times 16$ lattice at the same coupling. The eigenvectors associated with these modes were found to be localized and large just like on the $8^{3} \times 16$ lattice. There will be two competing effects as one goes to larger volume. As remarked, one will find more zero modes in the region $1.02<m<1.047$. But the effect of a single zero mode in larger volume will 
not be felt in as wide a region around the crossing point as in a smaller volume. This is because the number of modes that contribute to the pion propagator increase with volume and each single mode therefor has less weight.

Since for $\beta=5.7$ the gap does not reopen, the natural conclusion is that we are far from the continuum limit. We expect the gap to open again before $m=2$ if we go to a larger $\beta$. Tracing out the gapless region in the $(\beta, m)$ plane is necessary to probe massless quarks as one goes to the continuum limit. It is also important for a proper study of massless QCD using the overlap formalism [4] or the domain wall formalism [15]. For a proper study one has to work at a value of $\beta$ so that the gapless region ends before $m=2$. To reproduce the proper chiral behavior, the value of the mass that goes into the overlap Hamiltonian in (6), and the domain wall mass, should be kept greater than the upper bound of the gap $m_{2}(\beta)$. The gap also has to open before $m=2$ so that one can use level crossings for a proper measurement of the topological charge and topological susceptibility.

\section{Acknowledgements}

This research was supported by DOE contracts DE-FG05-85ER250000, DEFG05-96ER40979 and DE-FG03-96ER40956. R.L.S would like to thank Steve Sharpe and Larry Yaffe and R.G.E, U.M.H and R.N would like to thank Tony Kennedy, Tim Klassen and Stefan Sint for useful discussions. Computations were performed on the CM-2, workstation cluster and the new QCDSP supercomputer at SCRI.

\section{References}

[1] S. Gottlieb, Nucl. Phys. B53 (Proc. Suppl.) (1997) 155.

[2] T. Banks and A. Casher, Nucl. Phys. B169 (1980) 103.

[3] M. Atiyah and I. Singer, Ann. Math. 87 (1968) 484.

[4] R. Narayanan and H. Neuberger, Phys. Rev. Lett. 71 (1993) 3251; Nucl. Phys. B443 (1995) 305.

[5] P. Vranas and R. Narayanan, hep-lat/9702005, to appear in Nucl. Phys. B.

[6] K.M. Bitar, U.M. Heller and R. Narayanan, hep-th/9710052.

[7] W. Bardeen, A. Duncan, E. Eichten, G. Hockney and H. Thacker, heplat/9705008, hep-lat/9710084. 
[8] F. Butler, H. Chen, J. Sexton, A. Vaccarino and D. Weingarten, Nucl. Phys. B430 (1994) 179.

[9] B. Bunk, K. Jansen, M. Lüscher and H. Simma, DESY-Report (September 1994).

[10] T. Kalkreuter and H. Simma, Comput. Phys. Commun. 93 (1996) 33.

[11] K.-H. Mutter, Ph. De Forcrand, K. Schilling and R. Sommer, in Brookhaven 1986, Proceedings, Lattice Gauge Theory, '86, pg. 257.

[12] M.-C. Chu, J.M. Grandy, S. Huang and J.W. Negele, Phys. Rev. Lett. 70 (1993) 225; Phys. Rev. D48 (1993) 3340; Phys. Rev. D49 (1994) 6039; T.L. Ivanenko and J.W. Negele, hep-lat/9709130.

[13] G. 't Hooft, Phys. Rev. D14 (1976) 3432.

[14] B.-Sheikholeslami and R. Wohlert, Nucl. Phys. B259 (1985) 572.

[15] D.B. Kaplan, Phys. Lett. B288 (1992) 342, T. Blum and A. Soni, heplat/9611030; hep-lat/9706023; hep-lat/9710051. 